\title{
A Novel Approach on English Test Software Design Based on The.Net Framework
}

\author{
ZHANG Xiaoyu ${ }^{1, a}$ \\ ${ }^{1}$ Foreign Language School, Wuhan Polytechnic, Wuhan 430074, P.R China \\ azhangxiaoyu@126.com
}

Keywords: English Testing Software; .NET Framework; Online Platform; System Optimization.

\begin{abstract}
With the rapid development of Internet and computer technology, modern education based on the Internet as main means has become the trend of the development of the education today. Although the domestic English testing system gains more and more attention but most universities and colleges' equipment downtime rate is calculated in manual mode. Even if some of the systems can realize on-time downtime equipment information collection but for the vast majority of the company it is not always possible. In this paper, we design and implement the .NET based software for the English testing tasks. We adopt the state-of-the-art open source library to undertake the task and the result shows that our approach is correct and feasible.
\end{abstract}

\section{Introduction}

With the rapid development of Internet and computer technology, modern education based on the Internet as main means, has become the trend of the development of the education today. Examination is an important part of education process, it not only can stimulate the learning enthusiasm of students, also can let the teacher know students to master knowledge and ideas have a definite object, improve the classroom efficiency. Teachers in colleges and universities is the main body of education implementing yuan quality of teachers directly affect the quality of school education from the teacher evaluation is an important measure of teacher management is directly related to the school education quality improvement and optimization of teachers from objective fairness to improve teachers' evaluation is to improve teachers' level of job of teaching of important premise to improve the quality of teaching. The process of foreign language learning and teaching is to construct the rules of a language system and the process of it involves the perception, memory, thinking, imagination and a series of intellectual factors. Therefore, analysis and grasp the teaching object of intelligence and personality traits is an important link of foreign language teaching. Electronic information is accessible to a huge population in the world. Mobiles have become so much common that we have started to use it in every sphere of life. Whether it is entertainment or education, we can see a very intense use of mobiles and we can consider them as a new personal computer. It doesn't mean that desktop computers are now useless, but the mobile devices market is growing fast. They are cheap, convenient because of their portability, and due to geo location often more useful than PC. Although the domestic education industry testing system gains more and more attention but most enterprise equipment downtime rate is calculated in manual mode. Even if some of the testing system can realize enterprise downtime equipment information collection, but for the vast majority of the company, a specific production line, production workshop and its product is particular, so the company is facing a problem, shut down equipment basic information, such as production line, the operation, the device type duplicate entries, entry information error, entry personnel workload big problems. The information in order to reduce equipment downtime entry strength, improve the efficiency and accuracy, this paper used repeated equipment downtime information such as product, process, equipment, product information and related constants data together, forming a comprehensive information of downtime. Records that is to say, multiple unit only need to select the comprehensive outage information, then system will fill in the corresponding constant data. Based on the information equipment that has the downtime with fast input system, net MVC platform can 
completely meet the requirements of equipment downtime information collection, bring convenience for data entry personnel, improve the efficiency of the staff. As prefaced in the introduction, in recent years web applications have gained significant ground against desktop applications, not only due to the fact that their accessibility is unlimited by space, but thanks to the advances made on the plan of their security as well. Based on recent studies, more and more people are opting to perform their purchases and do their banking online on a daily basis. In the figure 1, we visually illustrate the popularity of different coding languages adopted by the developers.

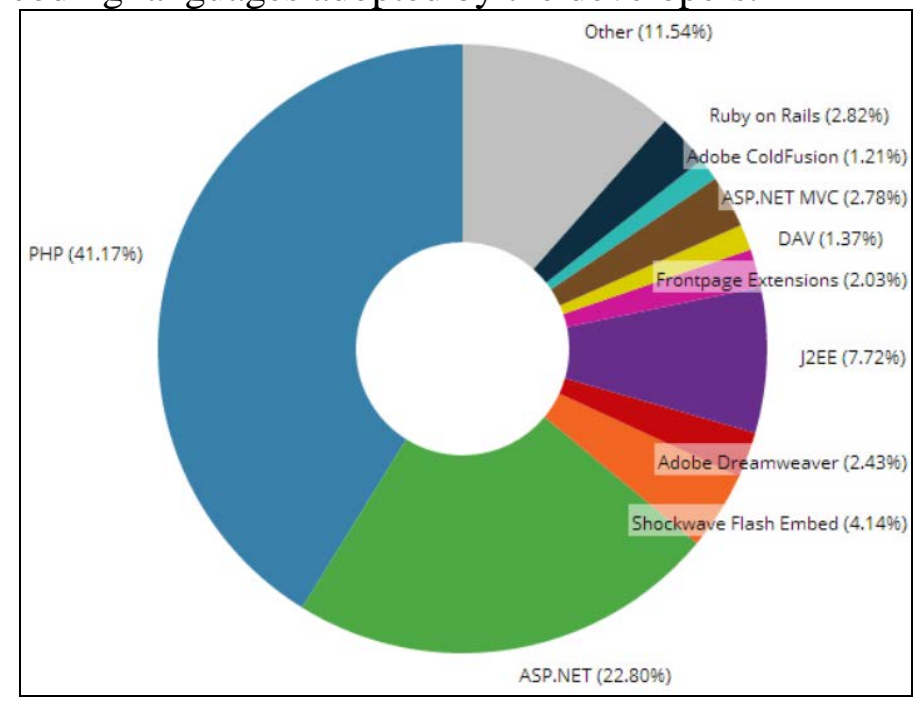

Fig. 1The Popularity of Different Programming Languages

In the traditional test methods, organization examination is a multistage process: teacher, print documents, organization examination of students, teachers, evaluation and examination paper analysis, statistical results and consume a large amount of manpower and material resources, in the paper, the work very is also prone to human error. But, as the questions and the number of students continue to increase, improve test, examination organization increasingly heavy workload, but also more and more complex. Therefore, we conduct research on designing English testing software and online platform based on .NET framework in this paper. The organization of the software is shown and discussed in the following sections.

\section{The Implementation and Organization of the Software}

The Data Structure and Pattern. In the process of WEB development, access to the database is a very important link, especially in the B/S construction system, database operation is almost an indispensable operation. System on the basis of the production line, combining with the production line of products, operations, equipment, personnel access, by setting the entry line, so the entry personnel with different permissions can enter different information of downtime. Integrated outage information function, the user will be licensed products, operations, equipment and production lines, forming a comprehensive information of downtime. This system can automatically calculate sorting statistics of avoid artificial processing of large amounts of data may result in the error evaluation after the completion to evaluate the teachers' management personnel can make the evaluation results public or archive management more convenient and efficient. Compared with other Web technologies, ASP.NET can more quickly and more easily establish a flexible, secure and stable Web applications. ASP.NET is the next generation of ASP products, but not the ASP simple upgrade. It is the ASP had the very big enhancement in performance, not only make it easier for developers to create dynamic Web content, can also make it easier to create complex and stable Web application. The work process is shown in figure 2. 


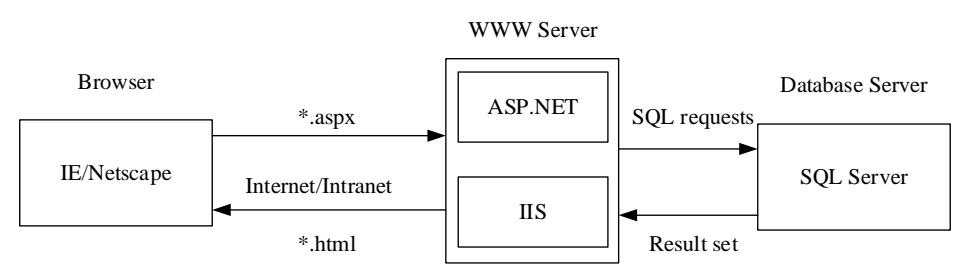

Fig. 2 The spatial data processing based on Web

In developed application, there is a feature to fetch data from database and display result in tabular format. For addressing these set of tasks in ASP.Net, combination of web controls, such as Label and TextBox can be used, however, there are data-bound controls such as the Grid View, Data Grid, Data List, Form View, Details View, Repeater, and Microsoft Char that be utilized and they are specific to this task. This is an important part of the data exchange between web and database technology. It is mentioned after processing EAUWE model from modeling program, source code automatically generated by traversing the tree structure. This section describes the method to traverse the tree, and code generation. As a first step of code generation some configuration data must be specified. These data include the name of the SQL server and database generated by the application to interact, and web application will be automatically generated path. It is an important bridge between the front-end and back-end database. In the figure 3, we show the structure of the framework.

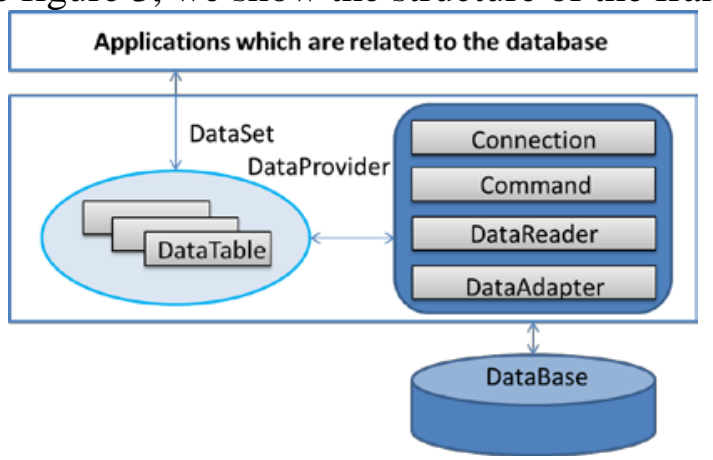

Fig. 3 The Structure of the .NET Framework

Implementation of the System. The decision of building the tree structure was motivated by the theoretical model of the abstract syntax tree (AST) and by its significant role in the source code generation process. However, the tree built during this study is very different from the AST, mainly because of the significant difference in the abstraction level. System provides special interfaces for each user, when a user name or password is not correct, the system page should be redirected to the login screen. The same, in every page, did the judgment on the legality of the user, when a user does not have the page operation permissions, the system page should be redirected to the login screen. In this way, it can effectively avoid the information leakage for the illegal users.

During the clarification of the content view description stage EAUWE profiles, it contains the classes, interfaces, abstract classes and enumeration. The source code is very obvious. Each generated text file contains a class, interface, or structure, proper name. It also contains all the appropriate "use" instruction, the namespace name, access modifiers, the actual content of elements. In addition to the enumeration of elements, properties of the NET-specific source code more sure of themselves, because the element does not contain the classical fields with the appropriate getter and setter methods, on the contrary, they have the property, two packaging method. On the function and the method of the specified circumstances, in the fifth part, the original source code can be specified in the modeling process. This is important, because it will cause a compiler error. In the automatically assigned key notes some attribute database. The fact that the motivation and put forward the following continuous passage. Although the user model of the element type with the content model, the main difference between them is, the user model elements in an application session, so they don't have any relational database web applications to interact. Therefore, these elements are not added to the database context in the final part. 


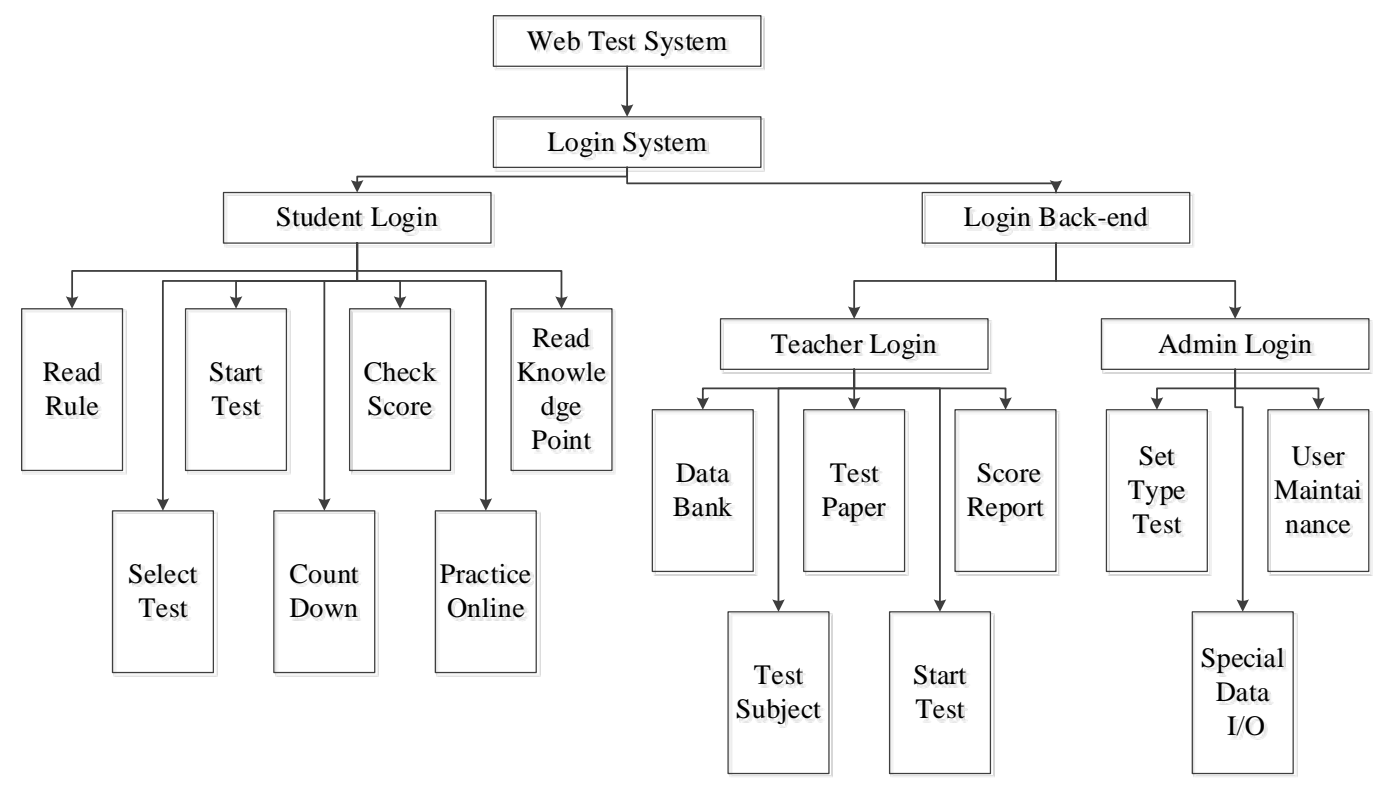

Fig. 4 The Implementation of the System

\section{Experiment and Code Demonstration}

In the figure 5, we show the label based code demonstration for the system, the organization and structure of the software is expressed in detail.

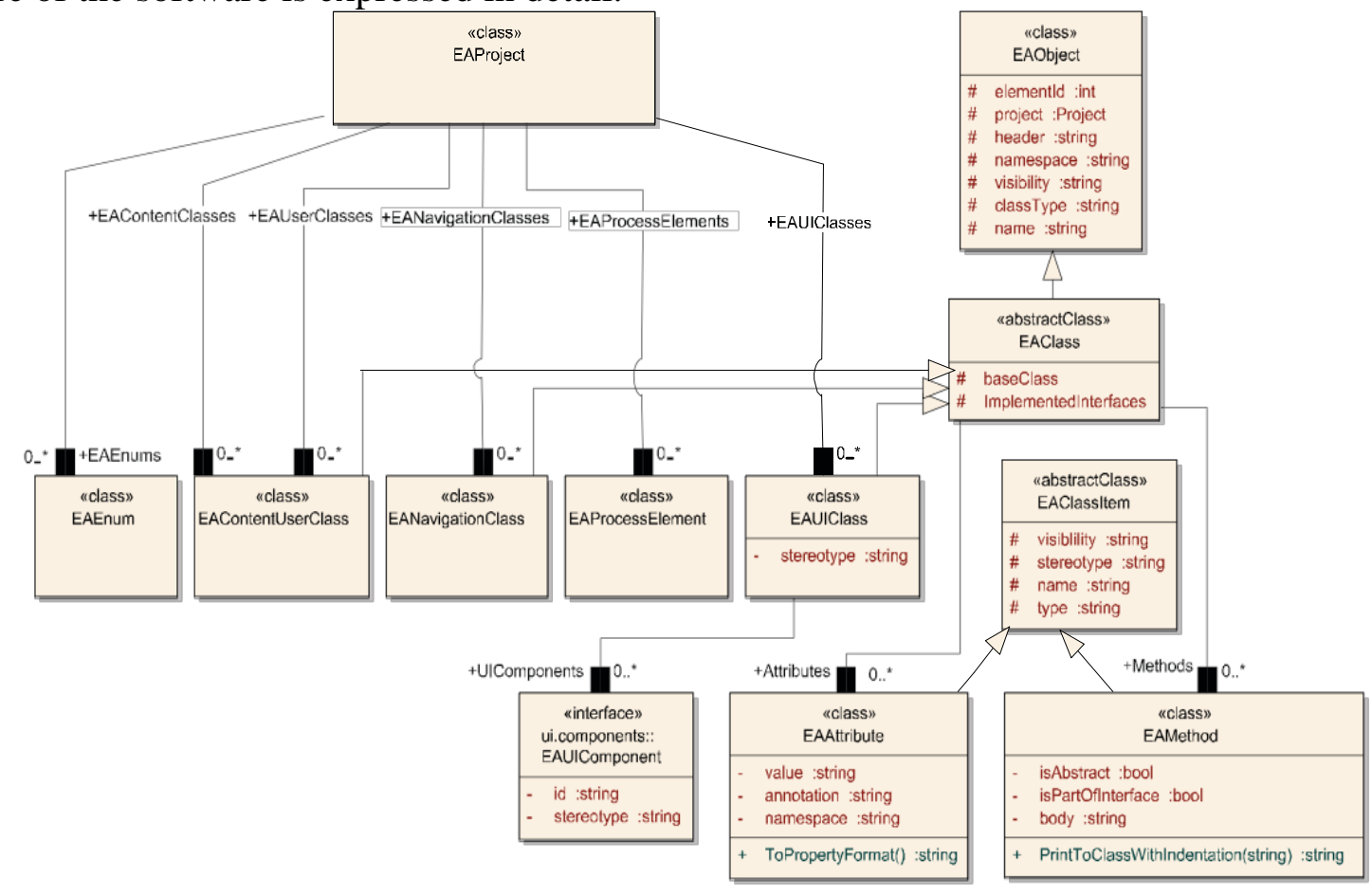

Fig. 5 The Code Structure Demonstration

\section{Conclusion and Summary}

With the rapid development of Internet and computer technology, modern education based on the Internet as main means, has become the trend of the development of the education today. In this paper, we design and implement the .NET based software for the English testing tasks. The code structure demonstration proves the effectiveness and efficiency of the proposed approach. In the future, we plan to add PHP language into the system to make it more robust. 


\section{References}

[1] *, M. L. T. (2014). The research and design of computerized adaptive english testing system. Advances in Applied Sciences Engineering \& Technology II.

[2] Loh, C. (2014). Nonlinear system identification of reinforced concrete steel structure: using pseudodynamic testing data. American Society of Civil Engineers, 130, 7, 836-847.

[3] Jain, A. (2014). Design, analysis, and full-scale testing of the rolled groove gasket joint system in awwa c303 bar-wrapped, steel-cylinder concrete pressure pipe. American Society of Civil Engineers, 4, 3, 234-243.

[4] Hongwu, L. (2014). How to improve the formative-assessment evaluation system in college english teaching. Journal of Jilin Agricultural Science \& Technology University.

[5] Koreeda, Y., Obata, S., Nishio, Y., Miura, S., Kobayashi, Y., \& Kawamura, K., et al. (2014). Development and testing of an endoscopic pseudo-viewpoint alternating system. International Journal of Computer Assisted Radiology \& Surgery. 